\title{
Comparison of Clock Solution of GLONASS Time Transfer Using ESOC Clock Products
}

\author{
Donghui $\mathrm{Yu}$ \\ Dept. of Software, Catholic University of Pusan, Korea \\ dhyu@cup.ac.kr
}

\begin{abstract}
GPS code measurements have been used to perform accurate and precise remote clock comparison, also called Time Transfer, and time synchronization. Initially, a technique based on Common View (CV) was used using a single-frequency for Time Transfer. Now, GPS Time Transfer uses dual-frequency measurements from geodetic receivers processed in All-in-View (AV). With the completion of the GLONASS constellation, GLONASS measurements could have been used in the dual-frequency AV mode. R2CGGTTS software is commonly used in GNSS (Global Navigation Satellite System) Time Transfer to provide the standard time transfer format, CGGTTS (Common GPS GLONASS Time Transfer), using observation files and navigation files written in RINEX format. Hence, the combination of GPS and GLONASS provides a clock solution based on AV mode using one time transfer solution. To provide the clock solution combined GPS and GLONASS measurements, the ISB (Inter System Bias) should be removed. One IGS analysis center, ESOC, provides satellite clocks for GPS as well as GLONASS satellites with the same reference for both constellations. Thanks to the ESOC clock products, to compute an AV solution combining the CGGTTS for both constellations is now possible.

In this paper, we present the results of the first step of the combination of GPS and GLONASS clock measurements, which is the clock correction of GLONASS code measurements using ESOC clock products.
\end{abstract}

Keywords: GNSS Time Transfer, CGGTTS, clock solution, Inter System Bias, GPS, GLONASS, code measurement

\section{Introduction}

\subsection{Overview of GNSS Time Transfer}

Time transfer is a scheme where multiple sites share a precise reference time. Time transfer solves problems such as astronomical observatories correlating observed flashes or other phenomena with each other, as well as cell phone towers coordinating handoffs as a phone moves from one cell to another [1].

Multiple techniques have been developed, often transferring reference clock synchronization from one point to another over long distances. Accuracy approaching one nanosecond worldwide is economically practical for many applications. Radio-based navigation systems are frequently used as time transfer systems.

In some cases, multiple measurements are made over a period of time, and exact time synchronization is determined retrospectively. In particular, time synchronization has been accomplished by using pairs of radio telescopes to listen to a pulsar, with the time transfer accomplished by comparing time offsets of the received pulsar signal.

There are four methods for time transfer. 
In a one-way time transfer method, one end system transmits its current time over some communication channel to one or more receivers. The receivers receive the time message and decode it, and either just report the time or adjust a local clock, which can provide holdover time reports in between the reception of messages. The advantage of one-way systems is that they can be technically simple and serve many receivers, as the transmitter is unaware of the receivers. The principal drawback of the one way time transfer system is that propagation delays of the communication channel remain uncompensated except in some advanced systems.

In a two-way time transfer method, the two peers will both transmit and will also receive each other's messages, thus performing two one-way time transfers to determine the difference between the remote clock and the local clock. The sum of these time differences is the round trip delay between the two nodes. It is often assumed that this delay is evenly distributed among the directions between the peers. Under this assumption, half the round-trip delay is the propagation delay to be compensated. A drawback is that the two-way propagation delay must be measured and used to calculate a delay correction. That function can be implemented in the reference source, in which case the source capacity limits the number of slaves that can be served, or by software in each slave.

In a Common-View (CV) method, the time difference between two clocks may be determined by simultaneously comparing each clock to a common reference signal that may be received at both sites. As long as both end stations receive the same satellite signal at the same time, the accuracy of the signal source is not important. In CV, the satellite clock (Sat1) is equally shown to both laboratories so that its errors are cancelled in the ground clock comparison equation in Figure 1. In CV, UTC (Lab1) - UTC (Lab2) equals to UTC (Lab1)-UTC (Sat1) - [UTC (Lab2)-UTC (Sat1)], but CV should be conducted for the same satellite and is limited by the time transfer distance.

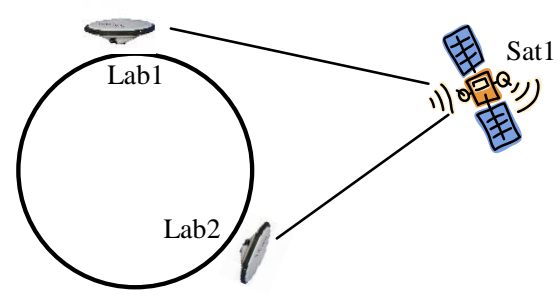

(a) GNSS CV time transfer

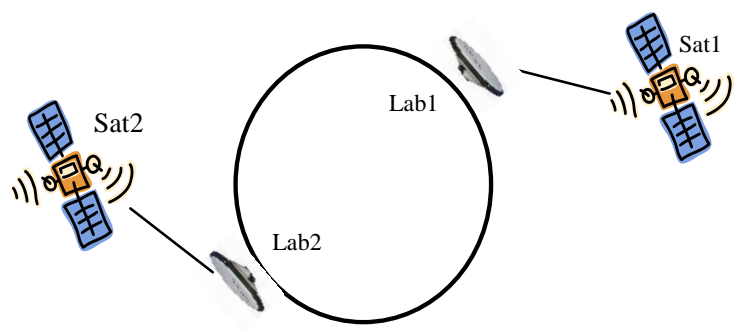

(b) GNSS AV time transfer

Figure 1. GNSS CV and AV Time Transfers for TAI and UTC

In AV, UTC(Lab1)-UTC(Lab2) equals to [UTC[Lab1]-Sat1]-[UTC(Lab2)$\mathrm{UTC}($ Sat2) + [Sat1-Sat2] in Figure 1. There is no common-view condition and the precise satellite clock corrections [Sat1-Sat2] are provided by global GNSS analysis centres. AV allows accurate time transfers at any time and between any two points located anywhere on the Earth. AV is the major technique for UTC generation [2]. 


\subsection{GLONASS}

GLONASS (from GLObal Navigation Satellite System, GLN for short) is a satellite navigation system based on GLONASS time scale operated by the Russian Space Forces with the aim of providing real-time, all-weather, three-dimensional positioning, velocity measuring, and timing with a world-wide coverage. The completely deployed GLONASS constellation is composed of 24 satellites in three orbital planes in each plane. GLONASS is comparable to other GNSS: the United States' GPS, the Beidou of China, and the Galileo positioning system of the European Union [3].

A major difference between the GPS and GLONASS constellations is that all the GLONASS satellites do not transmit the same frequency, i.e. each GLONASS satellite transmits on a different frequency using a 15-channel frequency division multiple access (FDMA) technique. As a result, the signal delay in the receiver is different for each satellite group emitting a given frequency. These differential biases must be either determined by calibration or estimated in addition to the clock solution [4]

\subsection{Procedure of GNSS Time Transfer}

The International Atomic Time (TAI) is calculated by the Bureau International des Poids et Mesures (BIPM) using a set of 400 atomic clocks distributed in about 60 time laboratories in the world. Each laboratory sends their clock data written in CGGTTS (Common GPS GLONASS Time Transfer Standard) format to BIPM. BIPM computes the weighted mean as the TAI. The legal time scale, Coordinated Universal Time (UTC), is obtained by adding the leap seconds to TAI [5].

A leap second is a one-second adjustment that is occasionally applied to the Coordinated Universal Time (UTC) in order to keep its time of day close to the mean solar time, or UT1. Without such a correction, time reckoned by Earth's rotation drifts away from atomic time because of irregularities in the Earth's rate of rotation. Since this system of correction was implemented in 1972, 26 leap seconds have been inserted. The most recent on June 30, 2015 at 23:59:60 UTC and the next leap second will be inserted on December 31, 2016, at 23:59:60 UTC.

The GNSS time transfer is performed using clock offsets collected in a fixed format, called CGGTTS [6]. These clock offsets represent the differences (clockREF) between the local clock and the reference timescale of the GNSS.

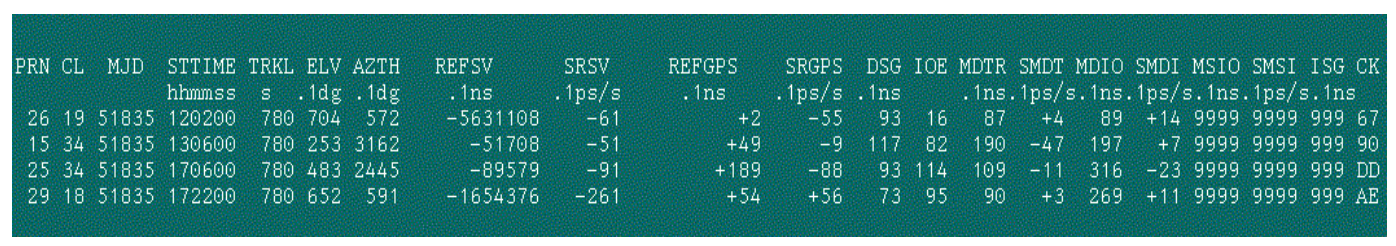

Figure 2. CGGTTS Result

Figure 2 shows the example of CGGTTS result and Table 1 presents the field explanation. 
Table 1. CGGTTS Field Explanation

\begin{tabular}{|c|c|}
\hline Field & Explanation \\
\hline PRN & the satellite vehicle PRN number \\
\hline $\mathrm{CL}$ & $\begin{array}{l}\text { the hexadecimal number that corresponds to the Common View class of } \\
\text { the track }\end{array}$ \\
\hline MJD & the five digit Modified Julian Date of the start of the track \\
\hline $\begin{array}{l}\text { STTIM } \\
\text { E }\end{array}$ & hour, minute, and second (in UTC) of the start of the track \\
\hline TRKL & the track length in seconds (a full track is 780 seconds) \\
\hline ELV & the elevation of the satellite at the midpoint of the track \\
\hline AZTH & the azimuth of the satellite at the midpoint of the track \\
\hline & $\begin{array}{l}\text { the time difference (measured in } .1 \text { nanosecond) between the laboratory } \\
\text { reference clock and satellite time, referred to the midpoint of the pass via a } \\
\text { linear fit }\end{array}$ \\
\hline SRSV & $\begin{array}{l}\text { the slope determined via the linear fit to produce REFSV (measured in } \\
.1 \text { picosecond per second) }\end{array}$ \\
\hline $\begin{array}{c}\text { REFGP } \\
S\end{array}$ & $\begin{array}{l}\text { the time differenc } \\
\text { reference clock and } \\
\text { via a linear fit }\end{array}$ \\
\hline SRGPS & $\begin{array}{l}\text { the slope determined via the linear fit to produce REFGPS (measured in } \\
.1 \text { picosecond per second) }\end{array}$ \\
\hline DSG & $\begin{array}{l}\text { the root mean square of the residuals of the linear fit used to produce } \\
\text { REFGPS }\end{array}$ \\
\hline IOE & $\begin{array}{l}\text { the index of ephemeris, a three digit decimal code indicating the } \\
\text { ephemeris used for computations }\end{array}$ \\
\hline MDTR & $\begin{array}{l}\text { the modeled tropospheric delay (measured in } .1 \text { nanosecond) referred to } \\
\text { the midpoint of the pass via a linear fit }\end{array}$ \\
\hline SMDT & $\begin{array}{l}\text { the slope determined via the linear fit to produce MDTR (measured in .1 } \\
\text { picosecond per second) }\end{array}$ \\
\hline MDIO & $\begin{array}{l}\text { the modeled ionospheric delay (measured in .1 nanosecond) referred to } \\
\text { the midpoint of the pass via a linear fit }\end{array}$ \\
\hline SMDI & $\begin{array}{l}\text { the slope determined via the linear fit to produce MDIO (measured in } .1 \\
\text { picosecond per second) }\end{array}$ \\
\hline MSIO & $\begin{array}{l}\text { the measured ionospheric delay (measured in .1 nanosecond) referred to } \\
\text { the midpoint of the pass via a linear fit, } 9999 \text { indicates no data available }\end{array}$ \\
\hline SMSI & $\begin{array}{l}\text { the slope determined via the linear fit to produce MSIO (measured in } .1 \\
\text { picosecond per second) }\end{array}$ \\
\hline ISG & $\begin{array}{l}\text { the root mean square of the residuals of the linear fit used to produce } \\
\text { MSIO }\end{array}$ \\
\hline $\mathrm{CK}$ & $\begin{array}{l}\text { the data line check sum in hexadecimal format, computed as the sum of } \\
\text { the ASCII values of the preceding } 115 \text { columns, modulo } 256\end{array}$ \\
\hline $\begin{array}{c}\text { optiona } \\
1\end{array}$ & $\begin{array}{l}\text { optional data may be presented beyond the check sum column, and is } \\
\text { not included in the CK value }\end{array}$ \\
\hline
\end{tabular}

They are obtained from the pseudorange measurements, which are calculated by the propagated time from a satellite to a receiver. There are several error factors while the satellite signal travels to a receiver. Figure 3 shows these error factors. There are the satellite clock error, the satellite orbit error, the tropospheric delay, the ionospheric delay, the multipath, the receiver clock error, the cable delay, the hardware delay, and so on [7]. 


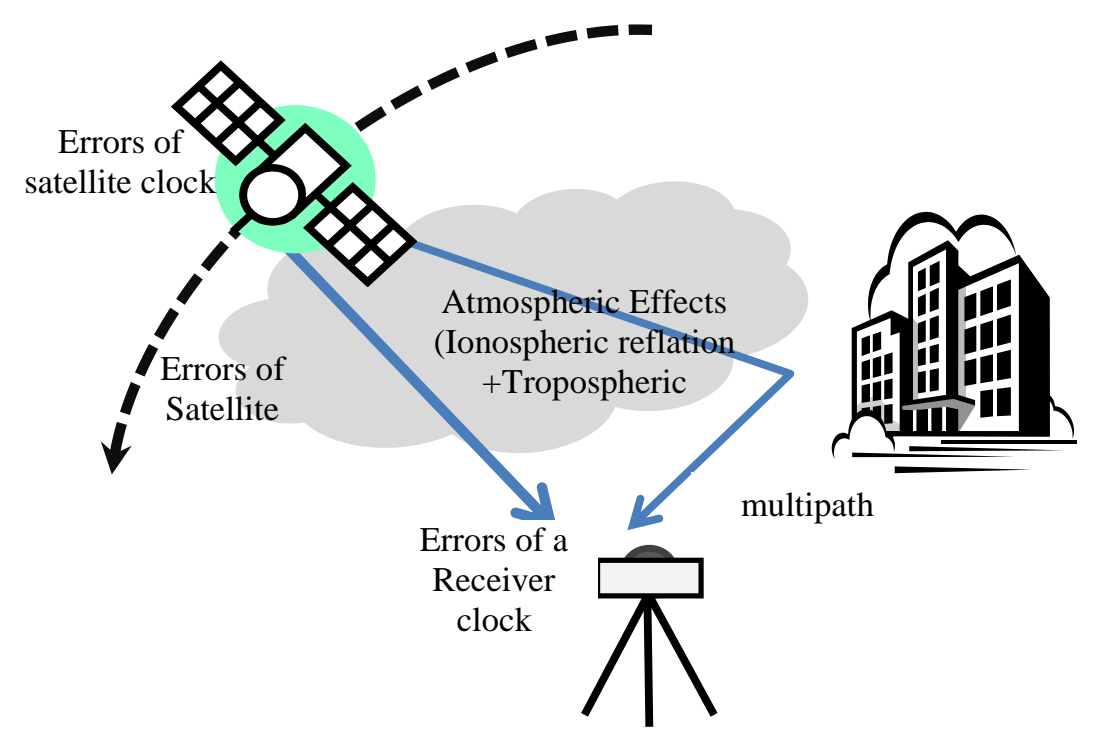

Input parameters : kind of Antenna, Hardware delay, cable delay, etc in paramCGGTTS.dat

Determination of satellite track to measure and Acquisition of 13 minutes measurements data in 16minutes interval in RINEX observation and navigation files

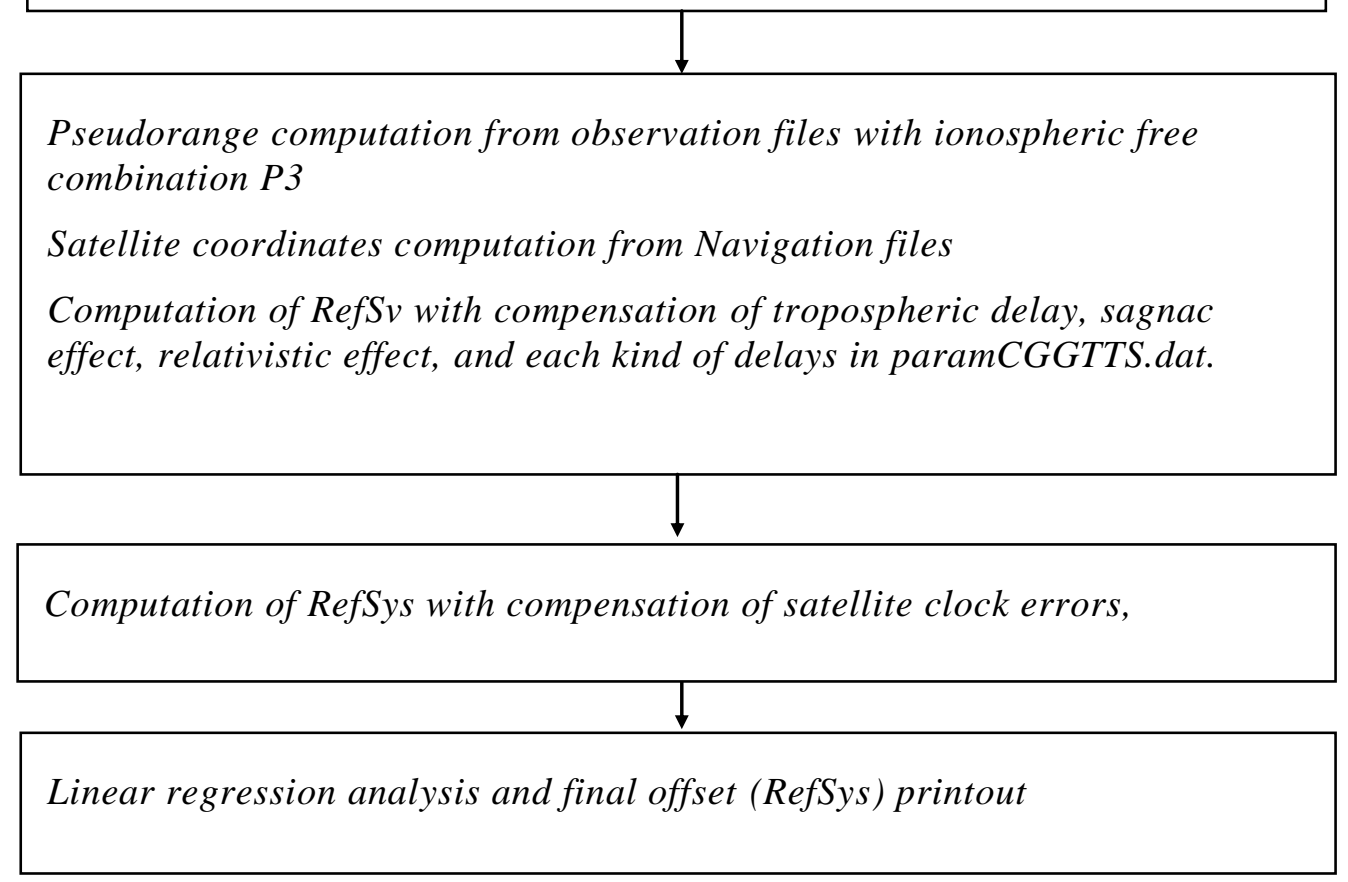

Figure 3. Procedure for Elimination of Delay Error Factors While GNSS Signal Is Travelled

A time correction must be performed because the observation files from geodetic receivers providing both GPS and GLONASS are dated in GPS time, while the reference of the GLONASS broadcast navigation message is GLONASS time, which is aligned on 
UTC. The two time scales GPS and GLONASS therefore differ by an integer number of leap seconds, the same as between GPS time and UTC.

Then a smoothing is performed over 13 minute observation tracks, and the results are then corrected for the instrumental hardware delays. These hardware delays correspond to the electric delays accumulated by the signal between the antenna phase center and the internal timing reference of the receiver. They are function of the frequency, so that the combination of measurements from satellites of different constellations requires taking into account the Inter-System Bias and Inter-Frequency Bias.

\section{Considerations of GLONASS Observations to Combine GPS Observations for Time Transfer}

As mentioned before, to combine GPS and GLONASS observations, Inter-system bias and Inter-Frequency bias should be processed. Inter-system bias is introduced as a time scale and time reference for each GNSS constellation.

As two constellations, GPS and GLONASS, use their own time scales, the leap seconds are corrected when the receiver computes the CGGTTS results.

Then, the results appearing in the CGGTTS files correspond to the differences between UTC (k) of time laboratory $\mathrm{k}$ and the reference of the broadcast satellite clocks. This reference is GPS time for GPS satellites and GLONASS time for the GLONASS satellites. In order to mitigate the errors due to the low precision of the broadcast satellite clocks and positions in CGGTTS results, the BIPM correct the CGGTTS results using more precise satellite orbits and clock product before the computation for TAI.

The GLONASS and GPS results obtained with the ionosphere-free combination P3 will be used together in a combined AV solution. To do this, the GPS and GLONASS results should be given with respect to the same reference.

We used the products delivered by one IGS analysis center, ESOC, which provides satellite clocks for GPS as well as GLONASS satellites with the same reference for both constellations. Hence, it is possible to compute an AV solution combining the CGGTTS results for satellites from both constellations [7]

ESOC has been provided a better stability to the biases on a daily basis from December 2012. In order to be consistent with the clock products used, which are computed on a daily and satellite basis, it was decided to compute the IFBs for each satellite and for each day separately from the CGGTTS results corrected with ESOC products [8].

\section{Comparison results for Clock Solution of GLONASS Time Transfer using ESOC Clock Products}

We compare the original clock offsets of GLONASS measurements in a CGGTTS results to the corrected clock offsets with the ESOC clock products. The correction is performed by applying the mid clock value in ESOC clock product for the scheduled track time to the clock offset, REFSYS, in the CGGTTS file. The CGGTTS files of 11 days are provided by KRISS (Korea Research Institute of Standards and Science). The observed dates are from MJD 57585(2016.7.16) to MJD 57595(2016.7.26).

REFSYS means the CGGTTS results, and SATSYS means the corrected results with ESOC clock product.

The average and standard deviations of clock solutions of 11 days are shown in Table 2 . 
Table 2. Statistics of 11 Days Clock Solutions Comparing the Original CGGTTS Results and Corrected Results with ESOC Clock Product

\begin{tabular}{|c|c|c|}
\hline & Average & Standard Deviation \\
\hline RefSYs & -489.628 & 90.96255 \\
\hline SatSys & -457.342 & 96.80912 \\
\hline
\end{tabular}

Figure 4 shows the comparison results of clock solutions of 11 days.

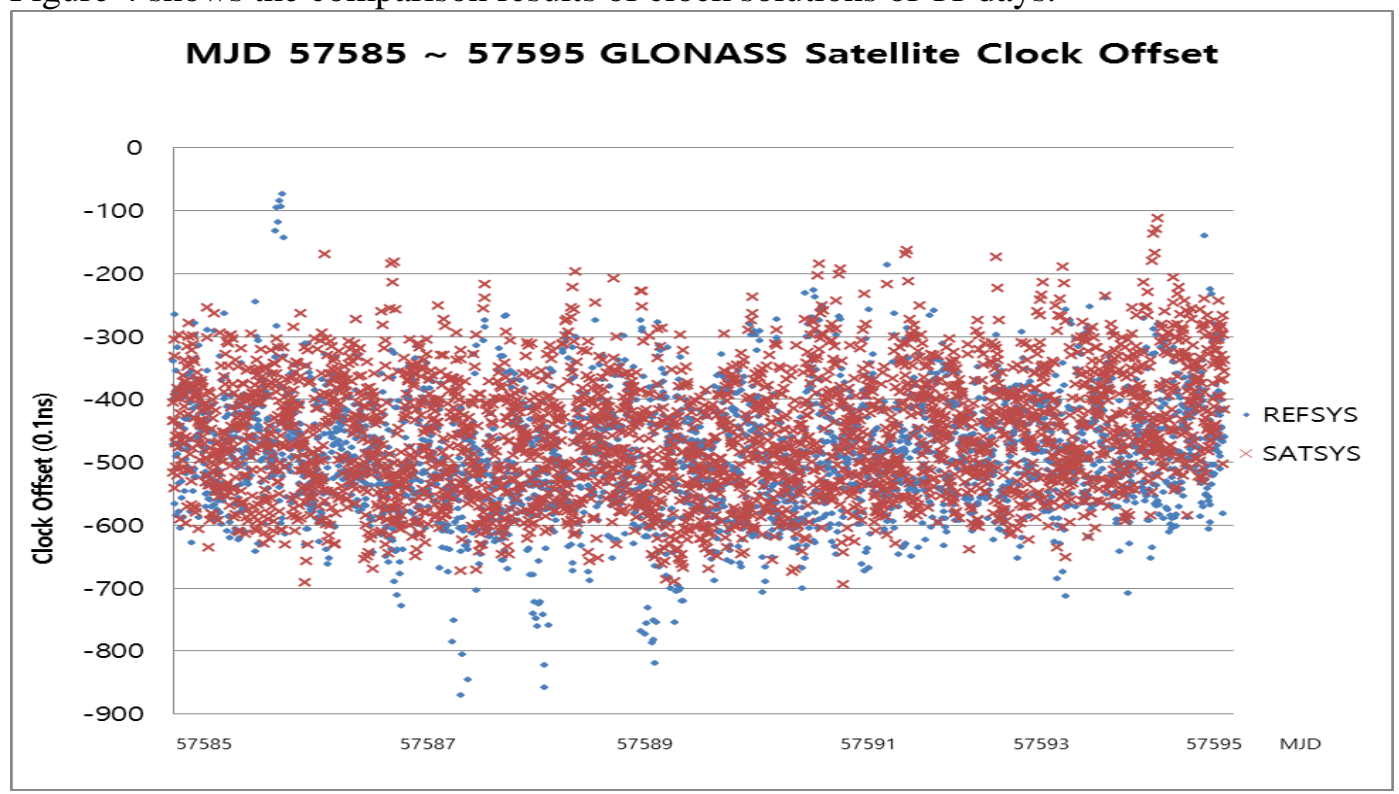

Figure 4. Results of 11 Days Clock Solutions

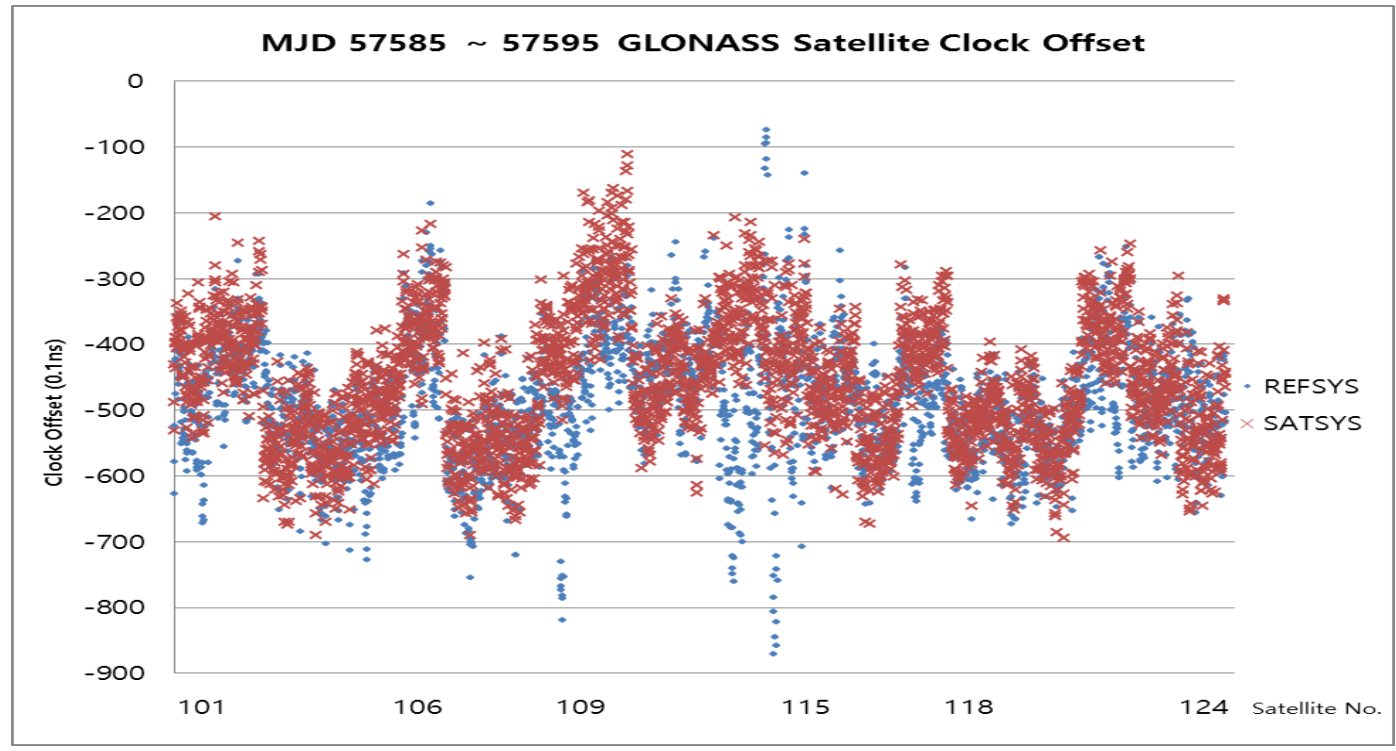

Figure 5. Results of 11 Days Clock Solutions for Each Satellite

Figure 6 shows the results of 11 days clock solutions for each satellite. Since the GLONASS satellites use FDMA technique, each satellite shows the different clock offset pattern. Significantly, satellite 109 and 115 show the large differences while satellite 124 
shows the similar pattern between the CGGTTS results (REFSYS) and corrected results (SATSYS). The detailed results are presented in Figure 6, 7, and 8.

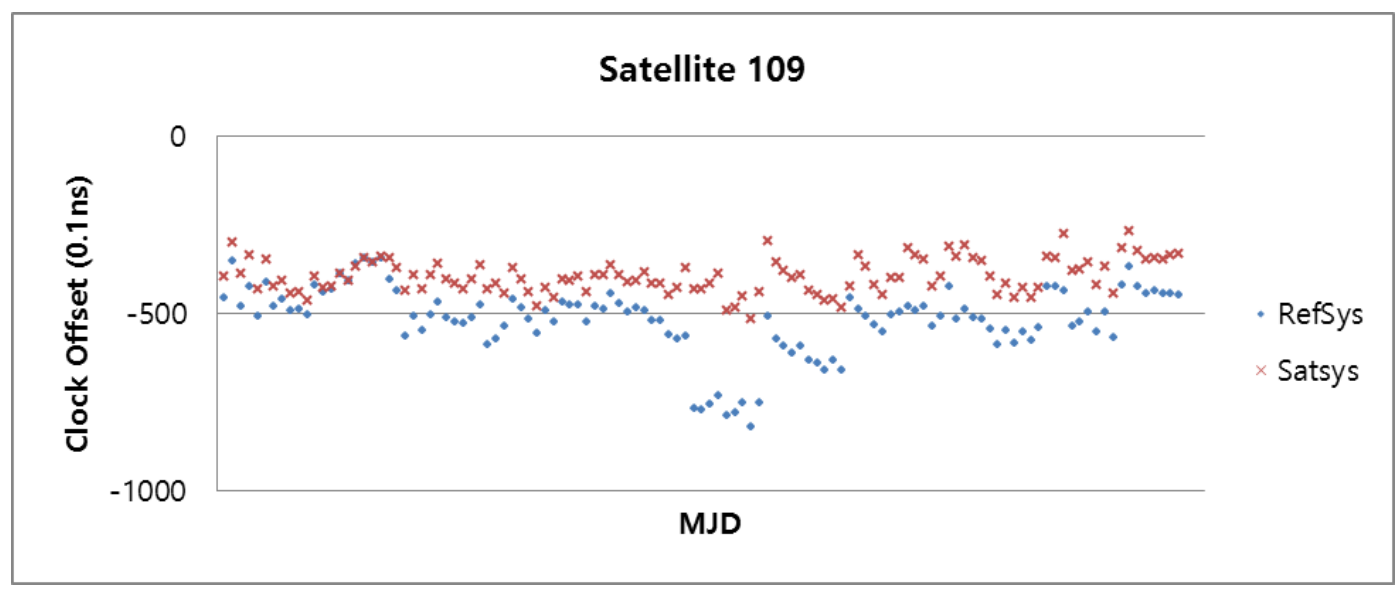

Figure 6. Results of 11 Days Clock Solutions for Satellites 109

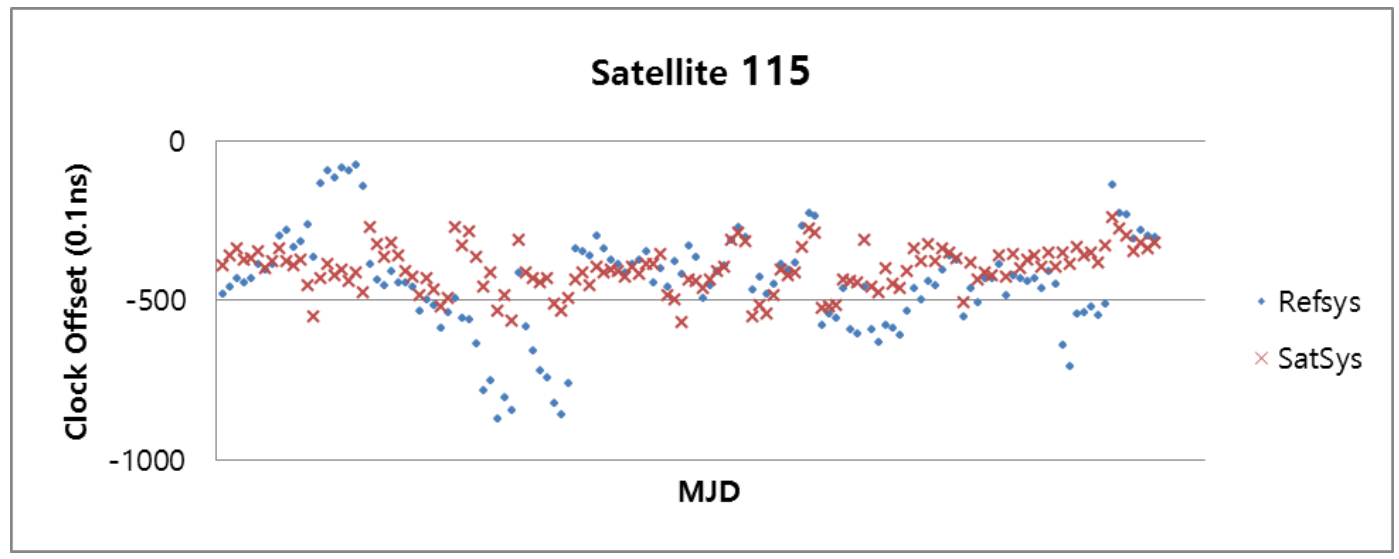

Figure 7. Results of 11 Days Clock Solutions for Satellites 115

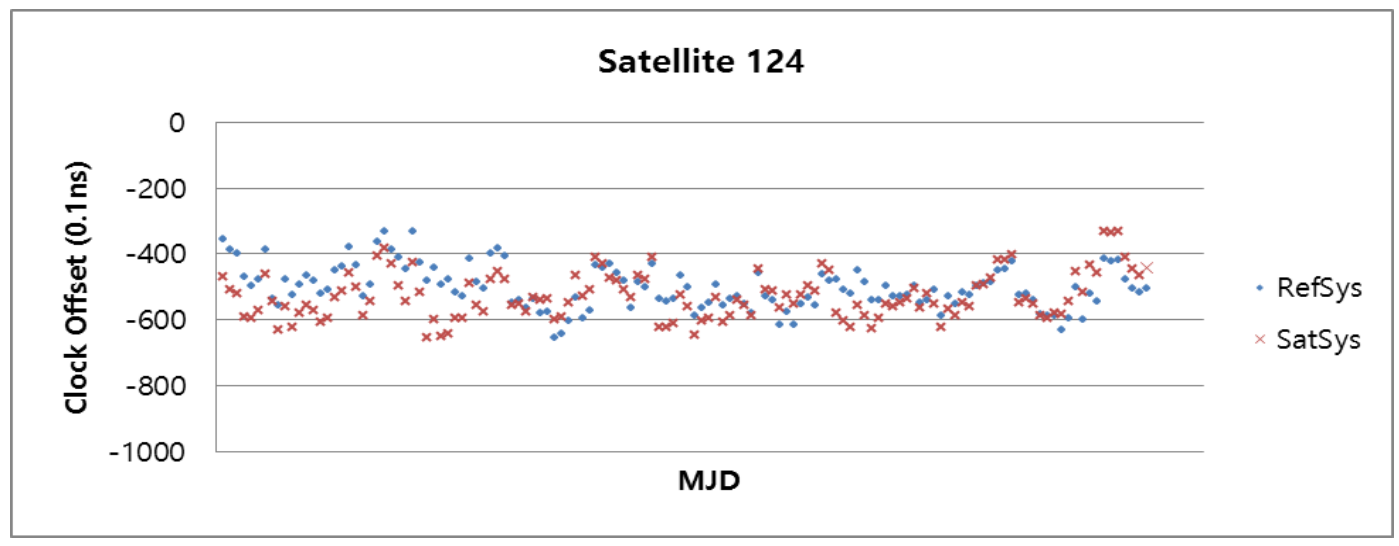

Figure 8. Results of 11 Days Clock Solutions for Satellites 124 
Figures 9 and 10 show the result of one day. According to the days, we can see that the clock solutions are different.

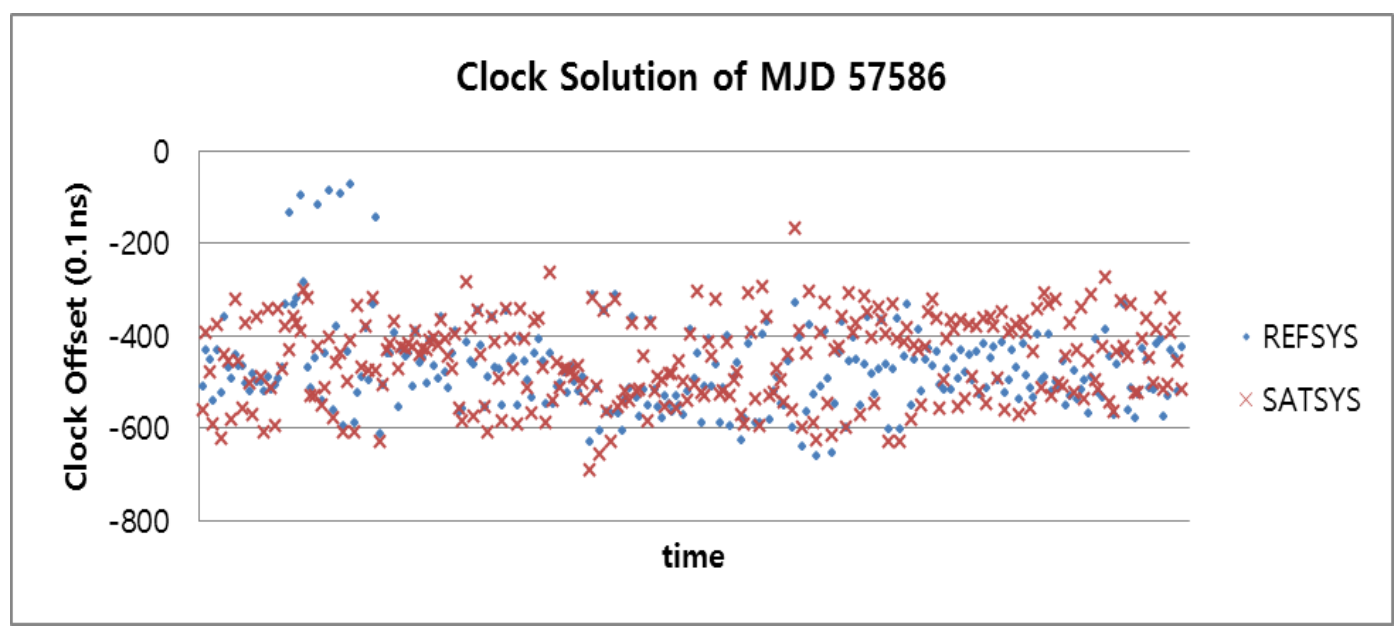

Figure 9. Results of MJD 57586 Clock Solutions for All Satellites

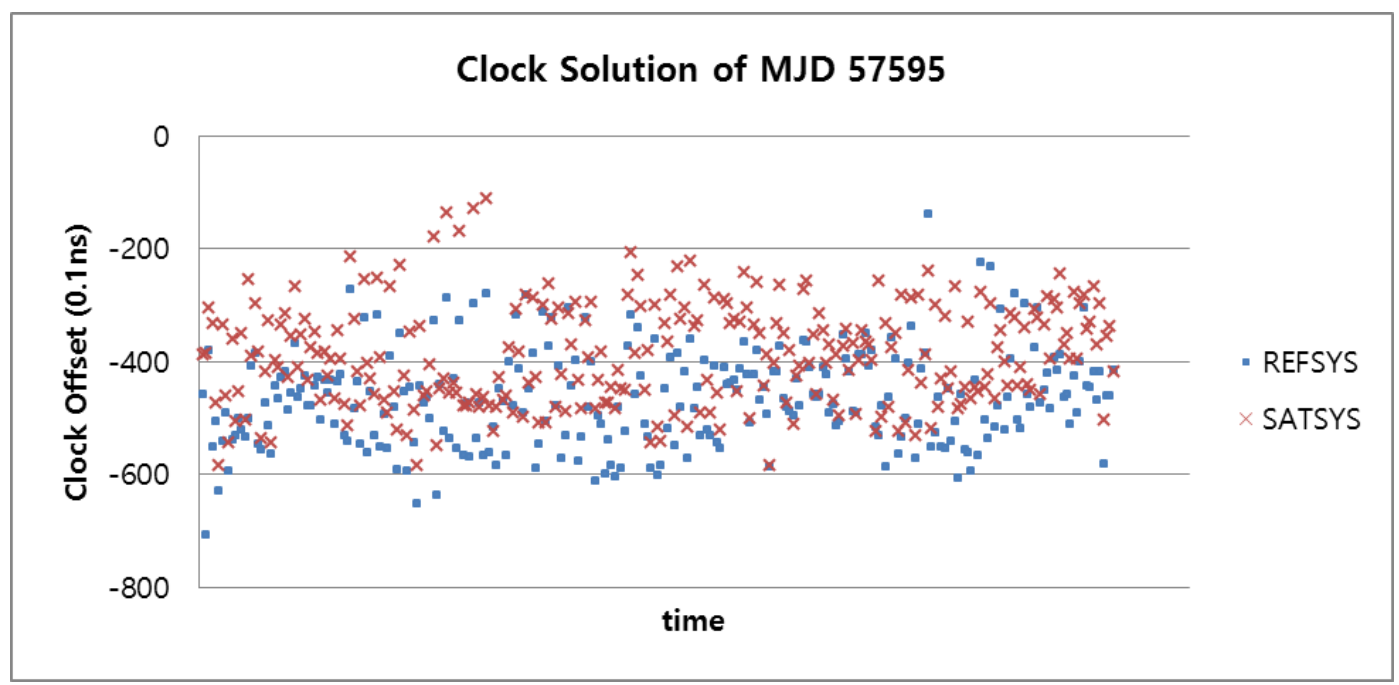

Figure 10. Results of MJD 57595 Clock Solutions for All Satellites

\section{Conclusion}

We present the results of the first step of the combination of GPS and GLONASS clock measurements, which is the clock correction of GLONASS code measurements using ESOC clock products, for generation of TAI. A further step in this use of GLONASS data will be to process the Inter Frequency Bias based on this result. 


\section{References}

[1] http://en.wikipedia.org

[2] D. Yu, Y. Lee, S. Yang, C. Lee and S.Yim, "Analysis of GLONASS Time Transfer with Korean Observation", in Information, vol. 17, (2014), pp.3405-3410.

[3] P. Defraigne, Q. Baire and A. Harmegnies, "Time and Frequency Transfer Combining GLONASS and GPS data", $42^{\text {nd }}$ Annual Precise Time and Time Interval(PTTI) meeting, pp. 263-274.

[4] P. Defraigne, "Multi-GNSS Time Transfer", in Journees 2013 Systemes de Reference Spatio-Temporels, (2013), pp. 109-114.

[5] Defraigne and C. Bruyninx, "Time Transfer for TAI using a geodetic receiver, An example with the Ashtech ZX11-T”, GPS solutions, (2001).

[6] J.Azoubib and W. Lewandowski, "CGGTTS GPS/GLONASS Data format version 02", in 7th CGGTTS meeting, (1998).

[7] G.Petit and Z. Jiang, "GPS All in View time transfer for TAI computation", in Metrologia, vol. 45, (2008), pp. 35-45.

[8] IGS products, [Internet] Available: http://igs.org/products/information.

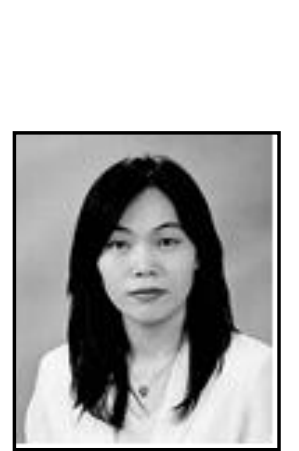

\section{Author}

Donghui Yu, she received her Doctor's degree in Computer Science in Pusan National University in 2001. Her research interests include time synchronization; GNSS time transfer and communication architecture 